Supporting Information for

\title{
Nafion-initiated ATRP of 1-vinylimidazole for Preparation of Proton Exchange Membranes
}

Kai Feng, $\uparrow$ Lei Liu, + Beibei Tang* $\uparrow$ Nanwen Li, + and Peiyi $\mathrm{Wu}^{*} \uparrow$

$\uparrow$ State Key Laboratory of Molecular Engineering of Polymers, Collaborative Innovation Center of Polymers and Polymer Composite Materials, Department of Macromolecular Science and Laboratory of Advanced Materials, Fudan University, Shanghai 200433, P. R. China.

$\$$ State Key Laboratory of Coal Conversion, Institute of Coal Chemistry, Chinese Academy of Sciences, Taiyuan, 030001, P. R. China

*E-mail: bbtang@fudan.edu.cn and peiyiwu@fudan.edu.cn. 


\section{- EXPERIMENTAL SECTION}

\section{Materials}

$5 \mathrm{wt} \%$ Nafion solution $(\mathrm{EW}=1100)$ was received from Sigma-Aldrich. Concentrated sulphuric acid, dimethylformamide (DMF) and $\mathrm{H}_{2} \mathrm{O}_{2}$ solution were provided by Sinopharm Chemical Reagent Co., Ltd. Dimethyl sulfoxide-d6 (D-DMSO), 1-vinylimidazole (VIm), bipyridyl and CuBr were purchased from Aladdin. All the other reagents were obtained from commercial suppliers and used as received.

\section{Membrane preparation}

The typical preparation procedures of the Nafion-PVIm membranes are as follows. First, certain amount of VIm monomer was dissolved in $10 \mathrm{~mL}$ as-received Nafion solution, and the weight ratio of VIm to Nafion polymer was controlled at $2: 1,4: 1$ or $8: 1$. The corresponding products were respectively marked as "Nafion-2PVIm", "Nafion-4PVIm" or "Nafion-8PVIm" in the following sections. After the addition of $0.06 \mathrm{~g}$ bipyridyl, the solution was degassed for three times through the typical freeze-pump-thaw processes. Second, $0.01 \mathrm{~g} \mathrm{CuBr}$ was added into the above solution and then the whole system was further degassed for another three times via the same freeze-pump-thaw processes. Third, the solution was vigorously stirred under $80{ }^{\circ} \mathrm{C}$ for $36 \mathrm{~h}$. Subsequently, the solution was dialyzed for at least 7 days to remove the catalyst and any residual VIm monomer, and then dried at $80{ }^{\circ} \mathrm{C}$ overnight. Fourth, the as-obtained product (i.e. Nafion-PVIm copolymer) was dissolved in DMF via sonication under $60{ }^{\circ} \mathrm{C}$. After that, the Nafion-PVIm/DMF solution was carefully cast onto a glass mould. It was dried in a vacuum oven by gradually increasing the temperature from 70 to $120{ }^{\circ} \mathrm{C}$ in $6 \mathrm{~h}$, and then further dried under vacuum at $120{ }^{\circ} \mathrm{C}$ overnight. 
Fifth, the Nafion-PVIm membrane was immersed in $3 \mathrm{wt} \% \mathrm{H}_{2} \mathrm{O}_{2}$ solution under $70{ }^{\circ} \mathrm{C}$ for $2 \mathrm{~h}$, and then immersed in $1 \mathrm{M} \mathrm{H}_{2} \mathrm{SO}_{4}$ solution at $80{ }^{\circ} \mathrm{C}$ for another $1 \mathrm{~h}$ to convert the membrane into $\mathrm{H}^{+}$ form. At last, the Nafion-PVIm membrane was rinsed by deionized $\mathrm{H}_{2} \mathrm{O}$ for several times before characterizations. The recast Nafion membrane was prepared via the same method as described above.

\section{General characterizations}

Nuclear magnetic resonance (NMR) spectra of the Nafion-PVIm copolymers and pristine Nafion polymer were obtained on a Varian Mercury plus $400 \mathrm{M}$ spectrometer with D-DMSO as the solvent and tetramethylsilane $\left({ }^{1} \mathrm{H}\right)$ and benzotrifluoride $\left({ }^{19} \mathrm{~F}\right)$ as the internal references. Before the NMR measurements, the Nafion-PVIm copolymers or pristine Nafion polymer were dissolved in D-DMSO at $140{ }^{\circ} \mathrm{C}$ for $\sim 48 \mathrm{~h}$ to give corresponding homogeneous solutions. Thermo gravimetric analyses (TGA) of the Nafion-PVIm and recast Nafion membranes were performed on a Perkin Elmer Thermal Analyzer at a heating rate of $10{ }^{\circ} \mathrm{C} \cdot \mathrm{min}^{-1}$ in $\mathrm{N}_{2}$ atmosphere. Attenuated total reflectance Fourier transform infrared spectroscopy (ATR-FTIR, with diamond as the crystal material) and transmission FTIR spectroscopy were characterized on a Nicolet Nexus 470 spectrometer with a resolution of $4 \mathrm{~cm}^{-1}$ and 64 scans. Before the FTIR measurement, the Nafion-PVIm and recast Nafion membranes were dried under $80{ }^{\circ} \mathrm{C}$ for $24 \mathrm{~h}$. The glass-transition temperatures of the recast Nafion and Nafion-2/4PVIm membranes were determined by a Mettler differential scanning calorimeter (DSC) in $\mathrm{N}_{2}$ at a heating rate of $20^{\circ} \mathrm{C} / \mathrm{min}$. Before the DSC analyses, all the samples were first heated from room temperature $\left(r . t\right.$.) to $220{ }^{\circ} \mathrm{C}$ to erase their previous thermal histories. Then, the temperature was decreased to $r . t$. to obtain the membrane samples in their standard states. The temperature resolution is $0.05{ }^{\circ} \mathrm{C}$. X-ray Photoelectron Spectroscopy (XPS) characterizations 
were carried out on Axis Ultra DLD XPS equipment (Kratos, England). Membrane morphology was characterized by a scanning electron microscopy (SEM, XL 30 ESEM-TMP PHILIP). All the samples were coated with gold before the SEM observation. The surface atomic force microscopy (AFM) measurement was conducted by a Multimode Nano 4 in a mechanical properties mode and a tapping mode. To reveal the microstructure differences between the Nafion-PVIm and recast Nafion membranes, X-ray diffraction (XRD) patterns were obtained on a PANalytical X'pert diffractometer with $\mathrm{Cu} \mathrm{K \alpha}$ radiation. The molecular weights of the Nafion-PVIm copolymers and pristine Nafion polymer were determined by a matrix assisted laser desorption ionization-time of flight / time of flight mass spectrometer (Maldi-TOF/TOF MS, AB SCIEX, 5800, America).

\section{Measurements of Water uptakes (WUs) ${ }^{1}$}

WUs were measured under three different conditions, i.e. 1) r. t., $100 \% \mathrm{RH}$; 2) $80{ }^{\circ} \mathrm{C}, 40 \% \mathrm{RH}$; 3) $80{ }^{\circ} \mathrm{C}, 20 \% \mathrm{RH}$. The typical procedures are as follow: First, $\mathrm{W}_{\text {dry }}$ was measured after the membrane was dried at $80{ }^{\circ} \mathrm{C}-0 \% \mathrm{RH}$ for $24 \mathrm{~h}$. Subsequently, the membrane was treated via three different ways: a) fully hydrated by being immersed in deionized $\mathrm{H}_{2} \mathrm{O}$ at room temperature for $24 \mathrm{~h}$. After that, the membrane was taken out and quickly sandwiched between two pieces of filter papers to remove the water remaining on the membrane surfaces.; or b) stored under $80{ }^{\circ} \mathrm{C}-40 \% \mathrm{RH}$ for $24 \mathrm{~h}$; or c) stored under $80{ }^{\circ} \mathrm{C}-20 \% \mathrm{RH}$ for $24 \mathrm{~h}$. Then, the membrane was immediately put into a sealed weighing bottle, and weighted (W). Thereby, WUs were calculated by the following equation:

$$
W U(\%)=\frac{W-W_{d r y}}{W_{d r y}} \cdot 100
$$

\section{Measurement of Proton conductivity ${ }^{1}$}

Proton conductivity was measured by a four-electrode method using AC impedance 
spectroscopy between $0.1 \mathrm{MHz}$ and $1 \mathrm{~Hz}$ with potentiostat control (CHI660d model). The Nafion-PVIm and recast Nafion membranes were equilibrated at the desired temperature and humidity for about $8 \mathrm{~h}$ before the measurement, and the environment was controlled by a temperature/humidity test chamber during the entire measurement. With respect to the time-dependent proton conductivity, the fully hydrated Nafion-PVIm and recast Nafion membranes were pre-treated under desired conditions for $30 \mathrm{~min}$ before the measurement.

\section{Measurement of methanol diffusivity ${ }^{1,2}$}

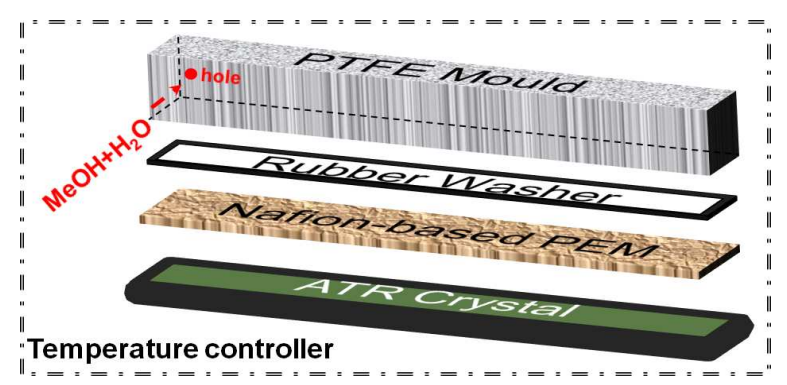

Figure S1. Schematic illustration of the home-made equipment used to characterize the methanol diffusivity of PEMs. ${ }^{1,2}$

First, the dry membrane was put onto the surface of the ZnSe crystal in the ATR cell (Nicolet Nexus 470 FTIR spectrometer) and then sandwiched between the ZnSe crystal and a PTFE mould (Figure S1). During the entire measurement, the membrane was always tightly attached onto the ZnSe crystal. ${ }^{2}$ Second, continuous FTIR data were collected as soon as $0.5 \mathrm{~mL}$ of $80 \mathrm{v} / \mathrm{v} \%$ methanol/ $\mathrm{H}_{2} \mathrm{O}$ solution was injected into the PTFE mould through a little hole. The hole was blocked during the entire measurement to avoid any evaporation of methanol/ $\mathrm{H}_{2} \mathrm{O}$. Using a macro program, the FTIR data, based on $4 \mathrm{~cm}^{-1}$ spectral resolution, were taken as a function of diffusion time with an acquisition time interval of $40 \mathrm{~s}$. Third, according to the increase in the peak area corresponding to the $-\mathrm{CH}_{3}$ stretching vibration, the Fickian diffusion equation (eq 1) put forward by Fieldson and 
Barbari $^{3}$ was used to estimate the diffusion coefficients of methanol of the Nafion-PVIm and recast Nafion membranes ${ }^{4}$.

$$
\frac{A_{t}}{A_{\infty}}=1-\frac{8 \gamma}{\pi[1-\exp (2 L \gamma)]} * \sum_{n=0}^{\infty}\left[\frac{\exp (g)\left[f \exp (-2 L \gamma)+(-1)^{n}(2 \gamma)\right]}{(2 n+1)\left(4 \gamma^{2}+f^{2}\right)}\right]
$$

Where $g=\frac{D(2 n+1)^{2} \pi^{2} t}{4 L^{2}} ; A_{t}$ is the absorbance are at time $t$, and $A_{\infty}$ is the equilibrium area; $L$ denotes the thickness of the PEM; $D$ refers to diffusion coefficient; $n$ and $\pi$ are constants.

The parameter $\gamma$ is defined as $\gamma=\frac{2 n_{2} \pi \sqrt{\sin ^{2} \theta-\left(\frac{n_{1}}{n_{2}}\right)^{2}}}{\lambda}$, where $\lambda$ is the wavelength of infrared beam in the ATR element; $\theta\left(\theta=45^{\circ} C\right)$ is the angle of the incidence of the infrared radiation; $n_{1}$ and $n_{2}$ are the refractive indexes of the PEM and the ATR crystal.

\section{Fabrication of Membrane/Electrode Assemblies (MEA) ${ }^{5}$}

The catalyst ink was prepared by mixing $40 \mathrm{wt} \% \mathrm{Pt} / \mathrm{C}$ catalyst (Johnson Matthey) with water, 1-propanal, and ionomoer solution ( $5 \mathrm{wt} \%$ Nafion in 1-propanal and water), and then homogenized for $30 \mathrm{~min}$ in an ultrasonic bath. To obtain a catalyst-coated membrane (CCM), the well-dispersed ink was sprayed onto the both sides of the Nafion-4PVIm membrane. The Pt loading and ionomer content measured by weighting in catalyst layer were both $0.5 \mathrm{mg} / \mathrm{cm}^{2}$. The MEA was fabricated by sandwiching two pieces of carbon paper and the CCM.

\section{Single Cell Test ${ }^{5}$}

The as-prepared MEA were assembled into a commercial fuel cell testing system (WonATech, South Korea) with an effective area of $5 \mathrm{~cm}^{2}$. Fuel cell test was conducted at $60{ }^{\circ} \mathrm{C}$ by passing fully humidified $\mathrm{H}_{2} / \mathrm{O}_{2}$ with the flow rate of $200 \mathrm{sccm}$. 


\section{- SUPPORTING RESULTS}

1.
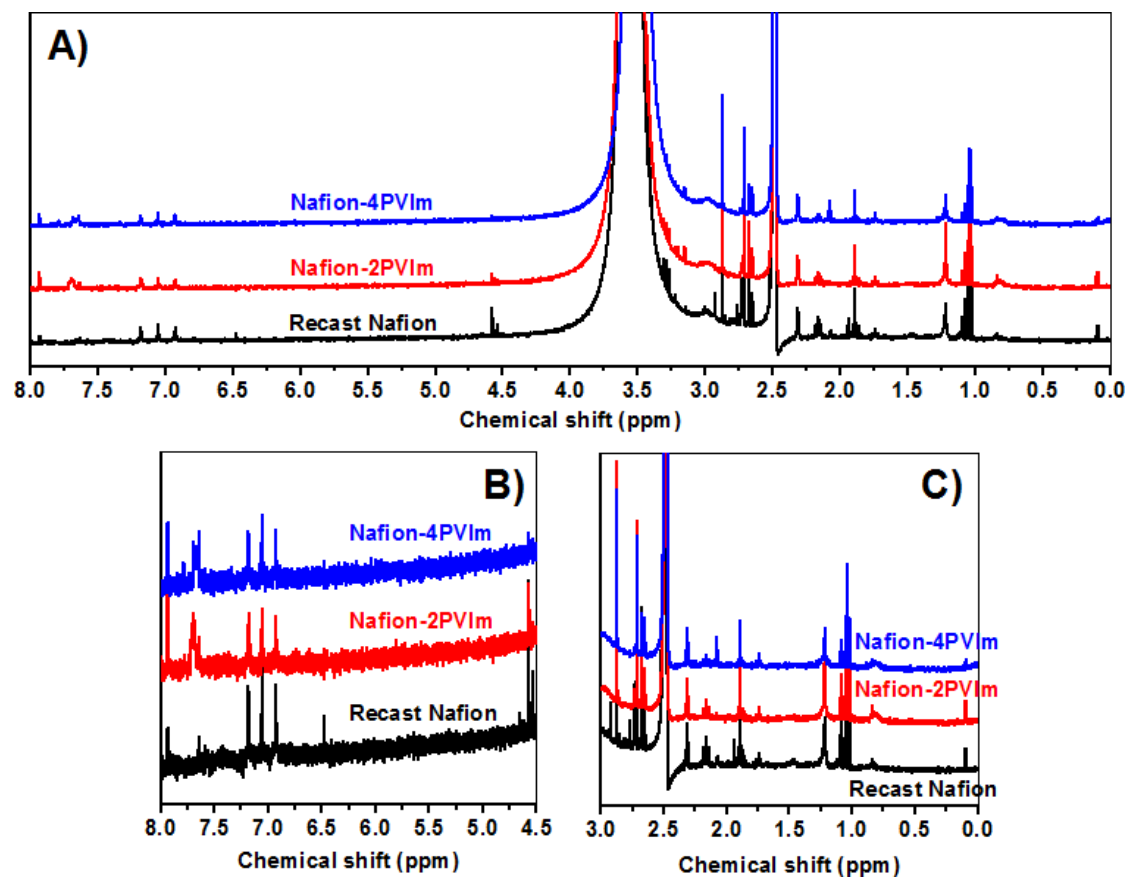

Figure S2. (A) ${ }^{1} \mathrm{H}$ NMR spectra of the recast Nafion and Nafion-2/4PVIm membranes (D-DMSO) in the chemical shift range of $0.0 \sim 8.0 \mathrm{ppm}$; Corresponding magnified spectra are shown in $(\mathrm{B} / \mathrm{C})$.

2.

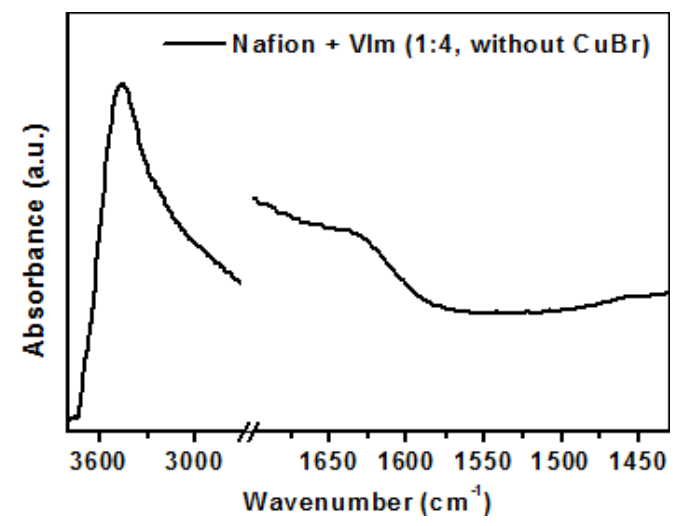

Figure S3. Transmission FTIR spectrum of the membrane prepared from the system of Nafion and VIm without the catalyst $\mathrm{CuBr}$. 
3.
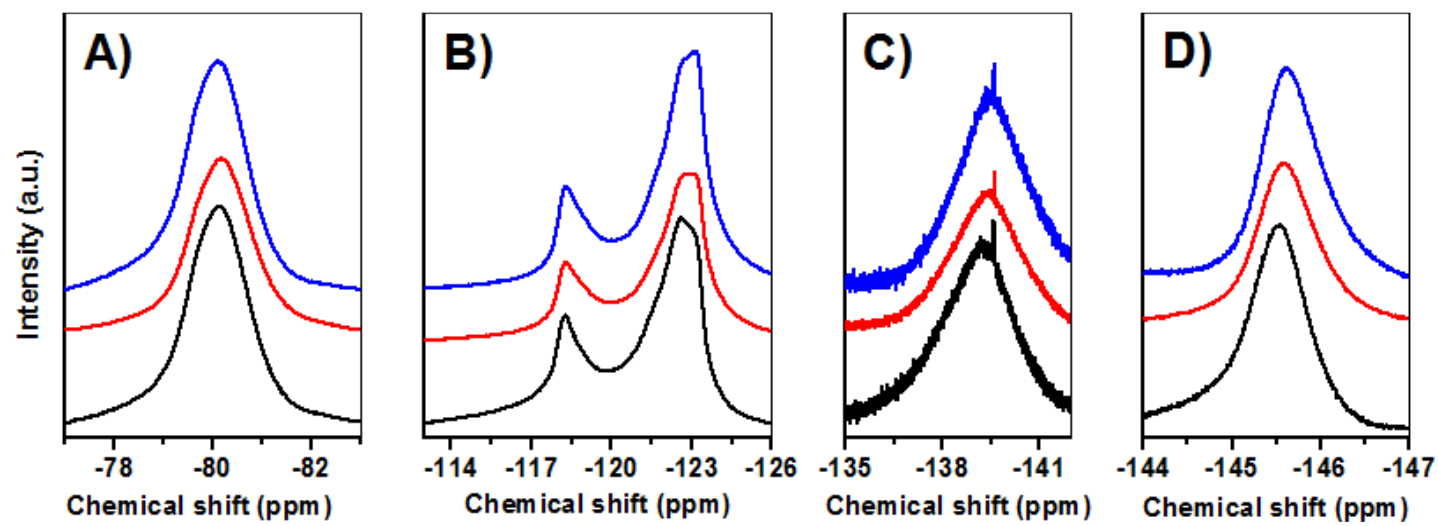

Figure S4. Four sets of the typical ${ }^{19}$ F NMR peaks of Nafion.

4.

Table S1. The integrated peak areas corresponding to the different C-F $\mathrm{F}_{(1 / 2 / 3)}$ groups of Nafion based on the ${ }^{19} \mathrm{~F}$ NMR data in Figure 2.

\begin{tabular}{|c|c|c|c|c|}
\hline & $2,2^{\prime}, 4,4^{\prime}, 5,5^{\prime}(\%)$ & $1,1^{\prime}, 7-9,7^{\prime}-9^{\prime}(\%)$ & $6,6^{\prime}(\%)$ & $3(\%)$ \\
\hline & (a.u.) & (a.u.) & (a.u.) & (a.u.) \\
\hline recast Nafion & 415840000 & 1247830000 & 34260400 & 59831100 \\
\hline Nafion-2PVIm & 331339000 & 1017460000 & 31130300 & 42538200 \\
\hline Nafion-4PVIm & 442124000 & 1368550000 & 41514600 & 54478500 \\
\hline
\end{tabular}

The typical four sets of ${ }^{19} \mathrm{~F}$ NMR peaks of Nafion are picked out from Figure 2, and their baselines are corrected with the aid of a software named as "The Unscrambler" (Figure S4). Subsequently, the peak area of each set of ${ }^{19} \mathrm{~F}$ NMR peak is integrated by "Origin 8.0". The data of the integrated peak areas are shown in Table S1. Take the " 2,2 ', 4, 4', 5, 5'" $\mathrm{F}$ atoms of the recast 
Nafion membrane for example, the corresponding relative ratio of the integrated peak area is estimated by dividing the total area $(415840000+1247830000+34260400+59831100)$ by 415840000. The other relative ratios are obtained via the same procedures, and results are shown in Table 1.

5.

A)

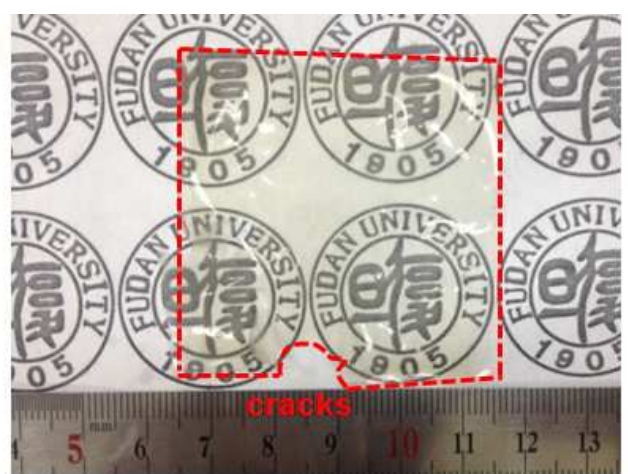

B)
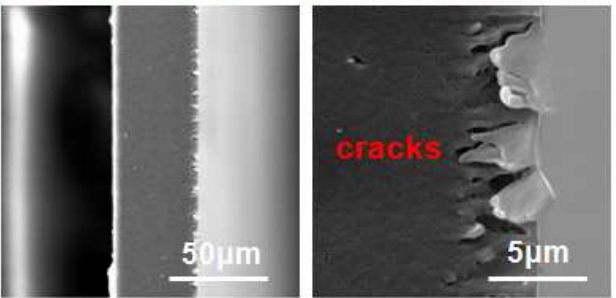

C)
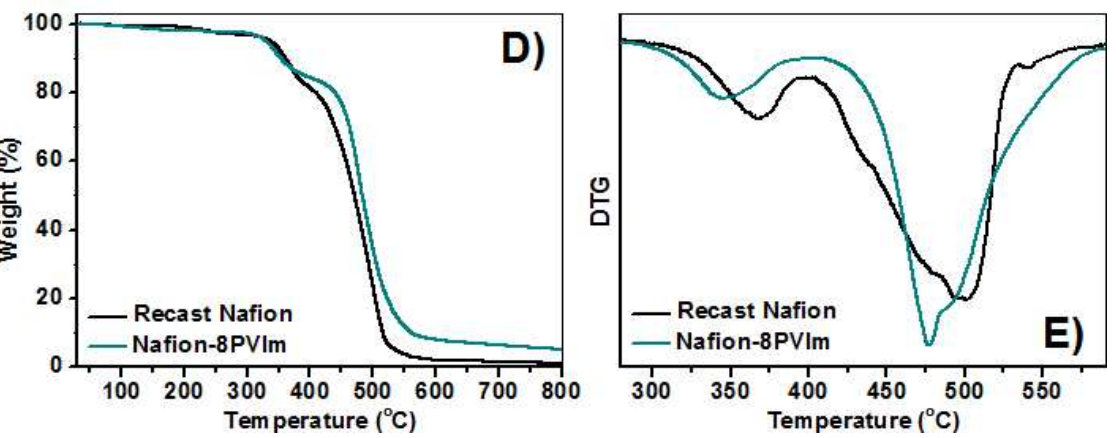

Figure S5. A digital photo (A), cross-sectional SEM images (B/C), TGA and DTG curves (D/E) of the Nafion-8PVIm membrane. 
6.

Table S2. Proton conductivity comparison between the Nafion-2/4PVIm membranes and other azoles-modified PEMs reported in the existing publications.

\begin{tabular}{|c|c|c|c|}
\hline PEMs & Proton conductivity $(\mathrm{S} / \mathrm{cm})$ & Conditions & References \\
\hline poly(1-vinylimidazole) + Nafion & 0.105 & r.t. $-100 \% \mathrm{RH}$ & 6 \\
\hline Nafion112 + 1H-1,2,4-triazole & $2.32 * 10^{-5}$ & \multirow{9}{*}{$180^{\circ} \mathrm{C}-0 \% \mathrm{RH}$} & \multirow{9}{*}{7} \\
\hline Nafion $115+1 \mathrm{H}-1,2,4$-triazole & $7.74 * 10^{-4}$ & & \\
\hline LNafion + 1H-1,2,4-triazole & $6.34 * 10^{-4}$ & & \\
\hline Nafion112 + 3-amino-1,2,4-triazole & $3.89 * 10^{-4}$ & & \\
\hline Nafion115 + 3-amino-1,2,4-triazole & $1.13 * 10^{-3}$ & & \\
\hline LNafion + 3-amino-1,2,4-triazole & $3.91 * 10^{-4}$ & & \\
\hline Nafion112 + 5-aminotetrazole & $1.06 * 10^{-5}$ & & \\
\hline Nafion $115+5$-aminotetrazole & $1.56^{*} 10^{-5}$ & & \\
\hline LNafion +5 -aminotetrazole & $1.56 * 10^{-4}$ & & \\
\hline \multirow{7}{*}{$\begin{array}{l}\text { Nafion } \\
\text { 2-propanoxymethylimidazole }\end{array}$} & 0.0015 & $80^{\circ} \mathrm{C}-0 \% \mathrm{RH}$ & \multirow{7}{*}{8} \\
\hline & 0.0013 & $90^{\circ} \mathrm{C}-0 \% \mathrm{RH}$ & \\
\hline & 0.0011 & $100^{\circ} \mathrm{C}-0 \% \mathrm{RH}$ & \\
\hline & 0.001 & $110^{\circ} \mathrm{C}-0 \% \mathrm{RH}$ & \\
\hline & 0.0014 & $120^{\circ} \mathrm{C}-0 \% \mathrm{RH}$ & \\
\hline & 0.0032 & $140^{\circ} \mathrm{C}-0 \% \mathrm{RH}$ & \\
\hline & 0.0048 & $160^{\circ} \mathrm{C}-0 \% \mathrm{RH}$ & \\
\hline $\begin{array}{l}\text { poly(ethylene glycol } \\
\text { dimethacrylate-co-vinylimidazole) }+ \\
\text { SPEEK }\end{array}$ & 0.036 & $30^{\circ} \mathrm{C}-100 \% \mathrm{RH}$ & 9 \\
\hline \multirow{6}{*}{$\begin{array}{l}\text { imidazole functionalized polyhedral } \\
\text { oligomeric silsesquioxane }+ \text { Nafion }\end{array}$} & 0.0008 & $40^{\circ} \mathrm{C}-0 \% \mathrm{RH}$ & \multirow{6}{*}{10} \\
\hline & 0.0017 & $60^{\circ} \mathrm{C}-0 \% \mathrm{RH}$ & \\
\hline & 0.0047 & $80^{\circ} \mathrm{C}-0 \% \mathrm{RH}$ & \\
\hline & 0.0098 & $100^{\circ} \mathrm{C}-0 \% \mathrm{RH}$ & \\
\hline & 0.017 & $120^{\circ} \mathrm{C}-0 \% \mathrm{RH}$ & \\
\hline & 0.022 & $140^{\circ} \mathrm{C}-0 \% \mathrm{RH}$ & \\
\hline
\end{tabular}


Table S2. (continued)

\begin{tabular}{|c|c|c|c|}
\hline PEMs & Proton conductivity (S/cm) & Conditions & References \\
\hline \multirow{9}{*}{$\begin{array}{l}\text { polysiloxane } r \text { bearing } \\
\text { 5-R-1H-tetrazoles and phosphoric } \\
\text { acid }\end{array}$} & 0.041 & $120^{\circ} \mathrm{C}-0 \% \mathrm{RH}$ & \multirow{9}{*}{11} \\
\hline & 0.039 & $110^{\circ} \mathrm{C}-0 \% \mathrm{RH}$ & \\
\hline & 0.038 & $100^{\circ} \mathrm{C}-0 \% \mathrm{RH}$ & \\
\hline & 0.038 & $90^{\circ} \mathrm{C}-0 \% \mathrm{RH}$ & \\
\hline & 0.036 & $80^{\circ} \mathrm{C}-0 \% \mathrm{RH}$ & \\
\hline & 0.032 & $70^{\circ} \mathrm{C}-0 \% \mathrm{RH}$ & \\
\hline & 0.0315 & $60^{\circ} \mathrm{C}-0 \% \mathrm{RH}$ & \\
\hline & 0.031 & $50^{\circ} \mathrm{C}-0 \% \mathrm{RH}$ & \\
\hline & 0.031 & $40^{\circ} \mathrm{C}-0 \% \mathrm{RH}$ & \\
\hline \multirow{8}{*}{ triazole modified POSS + Nafion } & 0.0024 & $40^{\circ} \mathrm{C}-0 \% \mathrm{RH}$ & \multirow{8}{*}{12} \\
\hline & 0.004 & $60^{\circ} \mathrm{C}-0 \% \mathrm{RH}$ & \\
\hline & 0.0085 & $80^{\circ} \mathrm{C}-0 \% \mathrm{RH}$ & \\
\hline & 0.011 & $100^{\circ} \mathrm{C}-0 \% \mathrm{RH}$ & \\
\hline & 0.015 & $120^{\circ} \mathrm{C}-0 \% \mathrm{RH}$ & \\
\hline & 0.017 & $140^{\circ} \mathrm{C}-0 \% \mathrm{RH}$ & \\
\hline & 0.003 & $150^{\circ} \mathrm{C}-0 \% \mathrm{RH}$ & \\
\hline & 0.0009 & $160^{\circ} \mathrm{C}-0 \% \mathrm{RH}$ & \\
\hline \multirow{8}{*}{$\begin{array}{l}\text { imidazole-functionalized nanosilica } \\
+ \text { Nafion }\end{array}$} & 0.09 & $25^{\circ} \mathrm{C}-100 \% \mathrm{RH}$ & \multirow{8}{*}{13} \\
\hline & 0.0964 & $30^{\circ} \mathrm{C}-100 \% \mathrm{RH}$ & \\
\hline & 0.1054 & $40^{\circ} \mathrm{C}-100 \% \mathrm{RH}$ & \\
\hline & 0.1131 & $50^{\circ} \mathrm{C}-100 \% \mathrm{RH}$ & \\
\hline & 0.1237 & $60^{\circ} \mathrm{C}-100 \% \mathrm{RH}$ & \\
\hline & 0.1354 & $70^{\circ} \mathrm{C}-100 \% \mathrm{RH}$ & \\
\hline & 0.1437 & $80^{\circ} \mathrm{C}-100 \% \mathrm{RH}$ & \\
\hline & 0.1589 & $90^{\circ} \mathrm{C}-100 \% \mathrm{RH}$ & \\
\hline \multirow{6}{*}{$\begin{array}{l}\text { imidazole functionalized } \\
\text { mesoporous silica }+ \text { Nafion }\end{array}$} & 0.005 & $40^{\circ} \mathrm{C}-0 \% \mathrm{RH}$ & \multirow{6}{*}{14} \\
\hline & 0.0062 & $60^{\circ} \mathrm{C}-0 \% \mathrm{RH}$ & \\
\hline & 0.007 & $80^{\circ} \mathrm{C}-0 \% \mathrm{RH}$ & \\
\hline & 0.0092 & $100^{\circ} \mathrm{C}-0 \% \mathrm{RH}$ & \\
\hline & 0.0107 & $130^{\circ} \mathrm{C}-0 \% \mathrm{RH}$ & \\
\hline & 0.0087 & $150^{\circ} \mathrm{C}-0 \% \mathrm{RH}$ & \\
\hline \multirow{5}{*}{ SPEEK + imidazole/($\left(\mathrm{HPO}_{3}\right)_{3}$} & 0.00055 & $55^{\circ} \mathrm{C}-0 \% \mathrm{RH}$ & \multirow{5}{*}{15} \\
\hline & 0.001 & $75^{\circ} \mathrm{C}-0 \% \mathrm{RH}$ & \\
\hline & 0.0016 & $95^{\circ} \mathrm{C}-0 \% \mathrm{RH}$ & \\
\hline & 0.0028 & $115^{\circ} \mathrm{C}-0 \% \mathrm{RH}$ & \\
\hline & 0.0038 & $135^{\circ} \mathrm{C}-0 \% \mathrm{RH}$ & \\
\hline
\end{tabular}


Table S2. (continued)

\begin{tabular}{|c|c|c|c|}
\hline PEMs & Proton conductivity ( $\mathrm{S} / \mathrm{cm})$ & Conditions & References \\
\hline \multirow{6}{*}{$\begin{array}{l}\text { SPEEK }+ \text { imidazole-type ionic } \\
\text { liquid }\end{array}$} & 0.0013 & $40^{\circ} \mathrm{C}-0 \% \mathrm{RH}$ & \multirow{6}{*}{16} \\
\hline & 0.0023 & $60^{\circ} \mathrm{C}-0 \% \mathrm{RH}$ & \\
\hline & 0.0042 & $80^{\circ} \mathrm{C}-0 \% \mathrm{RH}$ & \\
\hline & 0.0065 & $100^{\circ} \mathrm{C}-0 \% \mathrm{RH}$ & \\
\hline & 0.0076 & $120^{\circ} \mathrm{C}-0 \% \mathrm{RH}$ & \\
\hline & 0.0092 & $140^{\circ} \mathrm{C}-0 \% \mathrm{RH}$ & \\
\hline $\begin{array}{l}\text { poly(2-acrylamido-2-methyl-1-pro } \\
\text { panesulfonic } \\
\text { acid-co-5-(methacrylamido)tetrazol } \\
\text { e) }\end{array}$ & 0.009 & $150^{\circ} \mathrm{C}-0 \% \mathrm{RH}$ & 17 \\
\hline SPEEK + histidine & 0.00616 & r.t. $-100 \% \mathrm{RH}$ & 18 \\
\hline \multirow{5}{*}{$\begin{array}{l}\text { thermally } \\
\text { polybenzoxazole }\end{array}$} & 0.023 & $90^{\circ} \mathrm{C}-28 \% \mathrm{RH}$ & \multirow{5}{*}{19} \\
\hline & 0.038 & $100^{\circ} \mathrm{C}-28 \% \mathrm{RH}$ & \\
\hline & 0.064 & $110^{\circ} \mathrm{C}-28 \% \mathrm{RH}$ & \\
\hline & 0.105 & $120^{\circ} \mathrm{C}-28 \% \mathrm{RH}$ & \\
\hline & 0.16 & $130^{\circ} \mathrm{C}-28 \% \mathrm{RH}$ & \\
\hline \multirow{11}{*}{$\begin{array}{l}\text { phosphotungstic acid doped } \\
\text { polybenzimidazole decorated CNT } \\
+ \text { Nafion }\end{array}$} & 0.0889 & $25^{\circ} \mathrm{C}-100 \% \mathrm{RH}$ & \multirow{11}{*}{20} \\
\hline & 0.0954 & $30^{\circ} \mathrm{C}-100 \% \mathrm{RH}$ & \\
\hline & 0.1065 & $40^{\circ} \mathrm{C}-100 \% \mathrm{RH}$ & \\
\hline & 0.1237 & $50^{\circ} \mathrm{C}-100 \% \mathrm{RH}$ & \\
\hline & 0.1423 & $60^{\circ} \mathrm{C}-100 \% \mathrm{RH}$ & \\
\hline & 0.1637 & $70^{\circ} \mathrm{C}-100 \% \mathrm{RH}$ & \\
\hline & 0.1791 & $80^{\circ} \mathrm{C}-100 \% \mathrm{RH}$ & \\
\hline & 0.1902 & $90^{\circ} \mathrm{C}-100 \% \mathrm{RH}$ & \\
\hline & 0.2060 & $100^{\circ} \mathrm{C}-100 \% \mathrm{RH}$ & \\
\hline & 0.2123 & $110^{\circ} \mathrm{C}-100 \% \mathrm{RH}$ & \\
\hline & 0.2232 & $120^{\circ} \mathrm{C}-100 \% \mathrm{RH}$ & \\
\hline \multirow{4}{*}{$\begin{array}{l}\text { poly(vinyl alcohol)-sulfosuccinic } \\
\text { acid }+1 \mathrm{H}-1,2,4 \text {-triazole }\end{array}$} & 0.00154 & $140^{\circ} \mathrm{C}-0 \% \mathrm{RH}$ & \multirow{8}{*}{21} \\
\hline & 0.000404 & $140^{\circ} \mathrm{C}-0 \% \mathrm{RH}$ & \\
\hline & 0.00427 & $140^{\circ} \mathrm{C}-0 \% \mathrm{RH}$ & \\
\hline & 0.000332 & $140^{\circ} \mathrm{C}-0 \% \mathrm{RH}$ & \\
\hline \multirow{4}{*}{$\begin{array}{l}\text { poly(vinyl alcohol)-sulfosuccinic } \\
\text { acid + 3-amino-1,2,4-triazole }\end{array}$} & 0.00452 & $140^{\circ} \mathrm{C}-0 \% \mathrm{RH}$ & \\
\hline & 0.00138 & $140^{\circ} \mathrm{C}-0 \% \mathrm{RH}$ & \\
\hline & 0.00726 & $140^{\circ} \mathrm{C}-0 \% \mathrm{RH}$ & \\
\hline & 0.00133 & $140^{\circ} \mathrm{C}-0 \% \mathrm{RH}$ & \\
\hline
\end{tabular}


Table S2. (continued)

\begin{tabular}{|c|c|c|c|}
\hline PEMs & Proton conductivity $(\mathrm{S} / \mathrm{cm})$ & Conditions & References \\
\hline \multirow{11}{*}{$\begin{array}{l}\text { Nafion }+ \text { histidine modified carbon } \\
\text { nanotube }\end{array}$} & 0.091 & $25^{\circ} \mathrm{C}-100 \% \mathrm{RH}$ & \multirow{11}{*}{22} \\
\hline & 0.093 & $30^{\circ} \mathrm{C}-100 \% \mathrm{RH}$ & \\
\hline & 0.102 & $40^{\circ} \mathrm{C}-100 \% \mathrm{RH}$ & \\
\hline & 0.115 & $50^{\circ} \mathrm{C}-100 \% \mathrm{RH}$ & \\
\hline & 0.128 & $60^{\circ} \mathrm{C}-100 \% \mathrm{RH}$ & \\
\hline & 0.142 & $70^{\circ} \mathrm{C}-100 \% \mathrm{RH}$ & \\
\hline & 0.15 & $80^{\circ} \mathrm{C}-100 \% \mathrm{RH}$ & \\
\hline & 0.159 & $90^{\circ} \mathrm{C}-100 \% \mathrm{RH}$ & \\
\hline & 0.158 & $100^{\circ} \mathrm{C}-100 \% \mathrm{RH}$ & \\
\hline & 0.157 & $110^{\circ} \mathrm{C}-100 \% \mathrm{RH}$ & \\
\hline & 0.156 & $120^{\circ} \mathrm{C}-100 \% \mathrm{RH}$ & \\
\hline \multirow{11}{*}{$\begin{array}{l}\text { poly ((1-(4,4-diphenylether)-5-oxyb } \\
\text { enzimidazole)-benzimidazole })+ \\
\mathrm{BN}+\text { Nafion }\end{array}$} & 0.107 & $30^{\circ} \mathrm{C}-100 \% \mathrm{RH}$ & \multirow{11}{*}{23} \\
\hline & 0.131 & $40^{\circ} \mathrm{C}-100 \% \mathrm{RH}$ & \\
\hline & 0.140 & $50^{\circ} \mathrm{C}-100 \% \mathrm{RH}$ & \\
\hline & 0.148 & $60^{\circ} \mathrm{C}-100 \% \mathrm{RH}$ & \\
\hline & 0.157 & $70^{\circ} \mathrm{C}-100 \% \mathrm{RH}$ & \\
\hline & 0.166 & $80^{\circ} \mathrm{C}-100 \% \mathrm{RH}$ & \\
\hline & 0.174 & $90^{\circ} \mathrm{C}-100 \% \mathrm{RH}$ & \\
\hline & 0.188 & $100^{\circ} \mathrm{C}-100 \% \mathrm{RH}$ & \\
\hline & 0.097 & $110^{\circ} \mathrm{C}-100 \% \mathrm{RH}$ & \\
\hline & 0.074 & $120^{\circ} \mathrm{C}-100 \% \mathrm{RH}$ & \\
\hline & 0.028 & $130^{\circ} \mathrm{C}-100 \% \mathrm{RH}$ & \\
\hline \multirow{14}{*}{ 1H-1,2,3-benzotriazole + Nafion } & 0.0608 & $160^{\circ} \mathrm{C}-0 \% \mathrm{RH}$ & \multirow{14}{*}{24} \\
\hline & 0.0596 & $140^{\circ} \mathrm{C}-0 \% \mathrm{RH}$ & \\
\hline & 0.0579 & $130^{\circ} \mathrm{C}-0 \% \mathrm{RH}$ & \\
\hline & 0.0524 & $120^{\circ} \mathrm{C}-0 \% \mathrm{RH}$ & \\
\hline & 0.0451 & $110^{\circ} \mathrm{C}-0 \% \mathrm{RH}$ & \\
\hline & 0.0369 & $100^{\circ} \mathrm{C}-0 \% \mathrm{RH}$ & \\
\hline & 0.0302 & $90^{\circ} \mathrm{C}-0 \% \mathrm{RH}$ & \\
\hline & 0.0187 & $80^{\circ} \mathrm{C}-0 \% \mathrm{RH}$ & \\
\hline & 0.0136 & $70^{\circ} \mathrm{C}-0 \% \mathrm{RH}$ & \\
\hline & 0.0091 & $60^{\circ} \mathrm{C}-0 \% \mathrm{RH}$ & \\
\hline & 0.0082 & $50^{\circ} \mathrm{C}-0 \% \mathrm{RH}$ & \\
\hline & 0.0075 & $40^{\circ} \mathrm{C}-0 \% \mathrm{RH}$ & \\
\hline & 0.0071 & $30^{\circ} \mathrm{C}-0 \% \mathrm{RH}$ & \\
\hline & 0.0068 & $20^{\circ} \mathrm{C}-0 \% \mathrm{RH}$ & \\
\hline
\end{tabular}


Table S2. (continued)

\begin{tabular}{|c|c|c|c|}
\hline PEMs & Proton conductivity $(\mathrm{S} / \mathrm{cm})$ & Conditions & References \\
\hline \multirow{14}{*}{$\begin{array}{l}\text { benzimidazole tethered } \\
\text { perfluoropolymers } \\
\text { (perfluoroheptanoic acid) }+ \text { Nafion }\end{array}$} & 0.0863 & $30^{\circ} \mathrm{C}-30 \% \mathrm{RH}$ & \multirow{28}{*}{25} \\
\hline & 0.1054 & $30^{\circ} \mathrm{C}-40 \% \mathrm{RH}$ & \\
\hline & 0.1423 & $30^{\circ} \mathrm{C}-50 \% \mathrm{RH}$ & \\
\hline & 0.1738 & $30^{\circ} \mathrm{C}-60 \% \mathrm{RH}$ & \\
\hline & 0.2123 & $30^{\circ} \mathrm{C}-70 \% \mathrm{RH}$ & \\
\hline & 0.2726 & $30^{\circ} \mathrm{C}-80 \% \mathrm{RH}$ & \\
\hline & 0.3167 & $30^{\circ} \mathrm{C}-90 \% \mathrm{RH}$ & \\
\hline & 0.1054 & $80^{\circ} \mathrm{C}-30 \% \mathrm{RH}$ & \\
\hline & 0.1423 & $80^{\circ} \mathrm{C}-40 \% \mathrm{RH}$ & \\
\hline & 0.1827 & $80^{\circ} \mathrm{C}-50 \% \mathrm{RH}$ & \\
\hline & 0.2277 & $80^{\circ} \mathrm{C}-60 \% \mathrm{RH}$ & \\
\hline & 0.2726 & $80^{\circ} \mathrm{C}-70 \% \mathrm{RH}$ & \\
\hline & 0.3329 & $80^{\circ} \mathrm{C}-80 \% \mathrm{RH}$ & \\
\hline & 0.4494 & $80^{\circ} \mathrm{C}-90 \% \mathrm{RH}$ & \\
\hline \multirow{14}{*}{$\begin{array}{l}\text { benzimidazole } \\
\text { perfluoropolymers } \\
\text { (perfluorotetradecanoic } \\
\text { Nafion }\end{array}$} & 0.0639 & $30^{\circ} \mathrm{C}-30 \% \mathrm{RH}$ & \\
\hline & 0.0863 & $30^{\circ} \mathrm{C}-40 \% \mathrm{RH}$ & \\
\hline & 0.1076 & $30^{\circ} \mathrm{C}-50 \% \mathrm{RH}$ & \\
\hline & 0.1466 & $30^{\circ} \mathrm{C}-60 \% \mathrm{RH}$ & \\
\hline & 0.1738 & $30^{\circ} \mathrm{C}-70 \% \mathrm{RH}$ & \\
\hline & 0.2277 & $30^{\circ} \mathrm{C}-80 \% \mathrm{RH}$ & \\
\hline & 0.2865 & $30^{\circ} \mathrm{C}-90 \% \mathrm{RH}$ & \\
\hline & 0.0821 & $80^{\circ} \mathrm{C}-30 \% \mathrm{RH}$ & \\
\hline & 0.1108 & $80^{\circ} \mathrm{C}-40 \% \mathrm{RH}$ & \\
\hline & 0.1573 & $80^{\circ} \mathrm{C}-50 \% \mathrm{RH}$ & \\
\hline & 0.2019 & $80^{\circ} \mathrm{C}-60 \% \mathrm{RH}$ & \\
\hline & 0.2567 & $80^{\circ} \mathrm{C}-70 \% \mathrm{RH}$ & \\
\hline & 0.3012 & $80^{\circ} \mathrm{C}-80 \% \mathrm{RH}$ & \\
\hline & 0.4494 & $80^{\circ} \mathrm{C}-90 \% \mathrm{RH}$ & \\
\hline \multirow{6}{*}{ imidazole filler $\left(\mathrm{SiO}_{2}\right.$-Im $)+$ Nafion } & 0.01 & $120^{\circ} \mathrm{C}-20 \% \mathrm{RH}$ & \multirow{6}{*}{26} \\
\hline & 0.015 & $120^{\circ} \mathrm{C}-30 \% \mathrm{RH}$ & \\
\hline & 0.021 & $120^{\circ} \mathrm{C}-40 \% \mathrm{RH}$ & \\
\hline & 0.025 & $120^{\circ} \mathrm{C}-50 \% \mathrm{RH}$ & \\
\hline & 0.033 & $120^{\circ} \mathrm{C}-60 \% \mathrm{RH}$ & \\
\hline & 0.048 & $120^{\circ} \mathrm{C}-70 \% \mathrm{RH}$ & \\
\hline
\end{tabular}


Table S2. (continued)

\begin{tabular}{|c|c|c|c|}
\hline PEMs & Proton conductivity $(\mathrm{S} / \mathrm{cm})$ & Conditions & References \\
\hline \multirow{6}{*}{$\begin{array}{l}\mathrm{H}_{3} \mathrm{PO}_{4} \text {-dopped polybenzimidazole }+ \\
\mathrm{SiO}_{2} \text {-imidazole }\end{array}$} & 0.022 & $120^{\circ} \mathrm{C}-20 \% \mathrm{RH}$ & \multirow{6}{*}{27} \\
\hline & 0.03 & $120^{\circ} \mathrm{C}-30 \% \mathrm{RH}$ & \\
\hline & 0.041 & $120^{\circ} \mathrm{C}-40 \% \mathrm{RH}$ & \\
\hline & 0.05 & $120^{\circ} \mathrm{C}-50 \% \mathrm{RH}$ & \\
\hline & 0.06 & $120^{\circ} \mathrm{C}-60 \% \mathrm{RH}$ & \\
\hline & 0.07 & $120^{\circ} \mathrm{C}-70 \% \mathrm{RH}$ & \\
\hline $\begin{array}{l}\text { aluminophosphate-imidazole }+ \\
\text { Nafion }\end{array}$ & 0.1047 & r.t. $-100 \%$ RH & 28 \\
\hline \multirow{9}{*}{$\begin{array}{l}\text { cross-linked } \\
\text { triazole })+ \\
\text { acid) }\end{array}$} & 0.022 & $100^{\circ} \mathrm{C}-50 \% \mathrm{RH}$ & \multirow{9}{*}{29} \\
\hline & 0.018 & $80^{\circ} \mathrm{C}-50 \% \mathrm{RH}$ & \\
\hline & 0.014 & $60^{\circ} \mathrm{C}-50 \% \mathrm{RH}$ & \\
\hline & 0.0085 & $40^{\circ} \mathrm{C}-50 \% \mathrm{RH}$ & \\
\hline & 0.0015 & $20^{\circ} \mathrm{C}-50 \% \mathrm{RH}$ & \\
\hline & 0.00047 & $20^{\circ} \mathrm{C}-25 \% \mathrm{RH}$ & \\
\hline & 0.005 & $20^{\circ} \mathrm{C}-75 \% \mathrm{RH}$ & \\
\hline & 0.00062 & $60^{\circ} \mathrm{C}-25 \% \mathrm{RH}$ & \\
\hline & 0.055 & $60^{\circ} \mathrm{C}-75 \% \mathrm{RH}$ & \\
\hline \multirow{6}{*}{$\mathrm{SiO}_{2}-\mathrm{Im}+\mathrm{PBI}$} & 0.014 & $120^{\circ} \mathrm{C}-20 \% \mathrm{RH}$ & \multirow{6}{*}{30} \\
\hline & 0.0205 & $120^{\circ} \mathrm{C}-30 \% \mathrm{RH}$ & \\
\hline & 0.022 & $120^{\circ} \mathrm{C}-40 \% \mathrm{RH}$ & \\
\hline & 0.03 & $120^{\circ} \mathrm{C}-50 \% \mathrm{RH}$ & \\
\hline & 0.034 & $120^{\circ} \mathrm{C}-60 \% \mathrm{RH}$ & \\
\hline & 0.036 & $120^{\circ} \mathrm{C}-70 \% \mathrm{RH}$ & \\
\hline \multirow{12}{*}{ Nafion + imidazole } & 0.0247 & $40^{\circ} \mathrm{C}-70 \% \mathrm{RH}$ & \multirow{12}{*}{31} \\
\hline & 0.0302 & $50^{\circ} \mathrm{C}-70 \% \mathrm{RH}$ & \\
\hline & 0.0358 & $60^{\circ} \mathrm{C}-70 \% \mathrm{RH}$ & \\
\hline & 0.0408 & $70^{\circ} \mathrm{C}-70 \% \mathrm{RH}$ & \\
\hline & 0.0459 & $80^{\circ} \mathrm{C}-70 \% \mathrm{RH}$ & \\
\hline & 0.0562 & $90^{\circ} \mathrm{C}-70 \% \mathrm{RH}$ & \\
\hline & 0.0608 & $100^{\circ} \mathrm{C}-70 \% \mathrm{RH}$ & \\
\hline & 0.0639 & $110^{\circ} \mathrm{C}-70 \% \mathrm{RH}$ & \\
\hline & 0.0686 & $120^{\circ} \mathrm{C}-70 \% \mathrm{RH}$ & \\
\hline & 0.0721 & $130^{\circ} \mathrm{C}-70 \% \mathrm{RH}$ & \\
\hline & 0.0721 & $140^{\circ} \mathrm{C}-70 \% \mathrm{RH}$ & \\
\hline & 0.0728 & $150^{\circ} \mathrm{C}-70 \% \mathrm{RH}$ & \\
\hline
\end{tabular}


Table S2. (continued)

\begin{tabular}{|c|c|c|c|c|}
\hline PEMs & & Proton conductivity $(\mathrm{S} / \mathrm{cm})$ & Conditions & References \\
\hline \multirow{6}{*}{\multicolumn{2}{|c|}{ Nafion + 1,2,3-triazole }} & 0.0224 & $160^{\circ} \mathrm{C}-0 \% \mathrm{RH}$ & \multirow{13}{*}{32} \\
\hline & & 0.0166 & $140^{\circ} \mathrm{C}-0 \% \mathrm{RH}$ & \\
\hline & & 0.0067 & $120^{\circ} \mathrm{C}-0 \% \mathrm{RH}$ & \\
\hline & & 0.0050 & $100^{\circ} \mathrm{C}-0 \% \mathrm{RH}$ & \\
\hline & & 0.0025 & $80^{\circ} \mathrm{C}-0 \% \mathrm{RH}$ & \\
\hline & & 0.00007 & $60^{\circ} \mathrm{C}-0 \% \mathrm{RH}$ & \\
\hline \multirow{7}{*}{\multicolumn{2}{|c|}{ Nafion + benzimidazole }} & 0.0082 & $200^{\circ} \mathrm{C}-0 \% \mathrm{RH}$ & \\
\hline & & 0.0055 & $180^{\circ} \mathrm{C}-0 \% \mathrm{RH}$ & \\
\hline & & 0.0033 & $160^{\circ} \mathrm{C}-0 \% \mathrm{RH}$ & \\
\hline & & 0.0022 & $140^{\circ} \mathrm{C}-0 \% \mathrm{RH}$ & \\
\hline & & 0.0009 & $120^{\circ} \mathrm{C}-0 \% \mathrm{RH}$ & \\
\hline & & 0.0003 & $100^{\circ} \mathrm{C}-0 \% \mathrm{RH}$ & \\
\hline & & 0.00005 & $80^{\circ} \mathrm{C}-0 \% \mathrm{RH}$ & \\
\hline \multirow{8}{*}{$\begin{array}{l}\text { poly(vinylphosphonic } \\
\text { imidazole }\end{array}$} & & 0.0018 & $80^{\circ} \mathrm{C}-0 \% \mathrm{RH}$ & \multirow{8}{*}{33} \\
\hline & & 0.0025 & $90^{\circ} \mathrm{C}-0 \% \mathrm{RH}$ & \\
\hline & & 0.0037 & $100^{\circ} \mathrm{C}-0 \% \mathrm{RH}$ & \\
\hline & acid) & 0.0045 & $110^{\circ} \mathrm{C}-0 \% \mathrm{RH}$ & \\
\hline & & 0.005 & $120^{\circ} \mathrm{C}-0 \% \mathrm{RH}$ & \\
\hline & & 0.007 & $130^{\circ} \mathrm{C}-0 \% \mathrm{RH}$ & \\
\hline & & 0.006 & $140^{\circ} \mathrm{C}-0 \% \mathrm{RH}$ & \\
\hline & & 0.007 & $150^{\circ} \mathrm{C}-0 \% \mathrm{RH}$ & \\
\hline \multirow{6}{*}{ Nafion-2PVIm (ATRP) } & & 0.1403 & $100^{\circ} \mathrm{C}-40 \% \mathrm{RH}$ & \multirow{12}{*}{ This work } \\
\hline & & 0.008 & $80^{\circ} \mathrm{C}-20 \% \mathrm{RH}$ & \\
\hline & & 0.00956 & $90^{\circ} \mathrm{C}-20 \% \mathrm{RH}$ & \\
\hline & & 0.01149 & $100^{\circ} \mathrm{C}-20 \% \mathrm{RH}$ & \\
\hline & & 0.01337 & $110^{\circ} \mathrm{C}-20 \% \mathrm{RH}$ & \\
\hline & & 0.01397 & $120^{\circ} \mathrm{C}-20 \% \mathrm{RH}$ & \\
\hline \multirow{6}{*}{ Nafion-4PVIm (ATRP) } & & 0.2075 & $100^{\circ} \mathrm{C}-40 \% \mathrm{RH}$ & \\
\hline & & 0.01029 & $80^{\circ} \mathrm{C}-20 \% \mathrm{RH}$ & \\
\hline & & 0.01201 & $90^{\circ} \mathrm{C}-20 \% \mathrm{RH}$ & \\
\hline & & 0.01363 & $100^{\circ} \mathrm{C}-20 \% \mathrm{RH}$ & \\
\hline & & 0.01441 & $110^{\circ} \mathrm{C}-20 \% \mathrm{RH}$ & \\
\hline & & 0.01521 & $120^{\circ} \mathrm{C}-20 \% \mathrm{RH}$ & \\
\hline
\end{tabular}


7.

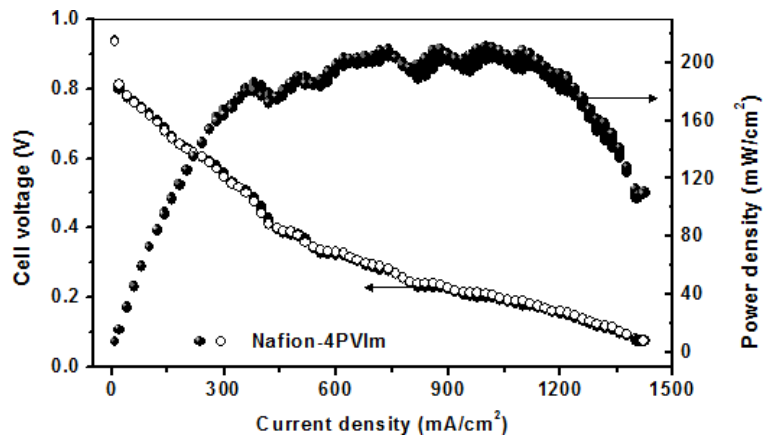

Figure S6. Polarization curve and power density curve of the PEMFC operated at $60{ }^{\circ} \mathrm{C}$ by using the Nafion-4PVIm membrane. Test conditions: $\mathrm{H}_{2} / \mathrm{O}_{2}$ flow rate of $200 \mathrm{~cm}^{3} / \mathrm{min}$; catalyst: $40 \% \mathrm{Pt} / \mathrm{C}$; Pt loading amount of $0.5 \mathrm{mg} / \mathrm{cm}^{2}$; ionomer concentration of $0.5 \mathrm{mg} / \mathrm{cm}^{2}$; humidifier temperature of 65 ${ }^{\circ} \mathrm{C}$; relative humidity of $100 \%$; active geometrical area: $5 \mathrm{~cm}^{2}$. 
8.

Table S3. Performance comparison of the FCs employing the Nafion-4PVIm membrane and the other azole-modified PEMs reported in the literatures.

\begin{tabular}{|c|c|c|c|c|c|}
\hline PEMs & $\begin{array}{l}\text { Applic } \\
\text { ations }\end{array}$ & Conditions & $\begin{array}{l}\text { Maximum } \\
\text { Power density } \\
\left(\mathbf{m W} / \mathrm{cm}^{2}\right)\end{array}$ & $\begin{array}{l}\text { Open cell } \\
\text { voltage }(\mathrm{V})\end{array}$ & $\begin{array}{l}\text { Referenc } \\
\text { es }\end{array}$ \\
\hline $\begin{array}{l}\text { poly(1-vinylimidazo } \\
\text { le) + Nafion }\end{array}$ & DMFC & $\begin{array}{l}2 \mathrm{M} \mathrm{MeOH} / \mathrm{H}_{2} \mathrm{O} \quad(5 \\
\mathrm{mL} / \mathrm{min}), \quad \mathrm{O}_{2} \quad(250 \\
\left.\mathrm{cm}^{3} / \mathrm{min}\right), 100 \% \mathrm{RH}\end{array}$ & $\sim 190$ & $\sim 0.60$ & 6 \\
\hline $\begin{array}{l}\text { Imidazole-modified } \\
\text { polyhedral } \\
\text { oligomeric } \\
\text { silsesquioxane + } \\
\text { Nafion }\end{array}$ & $\begin{array}{l}\text { PEMF } \\
\mathrm{C}\end{array}$ & $\begin{array}{l}\mathrm{H}_{2}(300 \mathrm{~mL} / \mathrm{min}), \mathrm{O}_{2} \\
(400 \quad \mathrm{~mL} / \mathrm{min}), \\
25 \% \mathrm{RH}, 120{ }^{\circ} \mathrm{C}\end{array}$ & $\sim 250$ & $\sim 0.95$ & 10 \\
\hline Nafion + imidazole & $\begin{array}{l}\text { PEMF } \\
\text { C }\end{array}$ & $60{ }^{\circ} \mathrm{C}$ & / & $\sim 0.55$ & 31 \\
\hline $\begin{array}{l}\text { Nafion } \\
1,2,3 \text {-triazole }\end{array}$ & $\begin{array}{l}\text { PEMF } \\
\mathrm{C}\end{array}$ & $\begin{array}{l}\mathrm{H}_{2}(100 \mathrm{~mL} / \mathrm{min}), \mathrm{O}_{2} \\
(300 \mathrm{~mL} / \mathrm{min}), 0 \% \mathrm{RH}, \\
100{ }^{\circ} \mathrm{C}\end{array}$ & / & $\sim 0.70$ & 32 \\
\hline $\begin{array}{l}\text { Nafion }+5 \mathrm{wt} \% \\
1,2,3 \text {-trizole }+66.7 \\
\mathrm{wt}^{2} \mathrm{H}_{3} \mathrm{PO}_{4}\end{array}$ & $\begin{array}{l}\text { PEMF } \\
\mathrm{C}\end{array}$ & $\begin{array}{l}\mathrm{H}_{2}(50 \mathrm{~mL} / \mathrm{min}), \mathrm{O}_{2} \\
(50 \mathrm{~mL} / \mathrm{min}), 0 \% \mathrm{RH}, \\
150{ }^{\circ} \mathrm{C}\end{array}$ & $\sim 35$ & $\sim 0.90$ & 34 \\
\hline Nafion-4PVIm & $\begin{array}{l}\text { PEMF } \\
\mathrm{C}\end{array}$ & $\begin{array}{l}\mathrm{H}_{2}(200 \mathrm{~mL} / \mathrm{min}), \mathrm{O}_{2} \\
(200 \quad \mathrm{~mL} / \mathrm{min}), \\
100 \% \mathrm{RH}, 60^{\circ} \mathrm{C}\end{array}$ & $\sim 210$ & $\sim 0.95$ & This work \\
\hline
\end{tabular}

\section{- REFERENCES}

(1) Feng, K.; Tang, B. B.; Wu, P. Y. Sulfonated Graphene Oxide-Silica for Highly Selective Nafion-based Proton Exchange Membranes. J. Mater. Chem. A 2014, 2, 16083-16092.

(2) Feng, K.; Tang, B. B.; Wu, P. Y. A " $\mathrm{H}_{2} \mathrm{O}$ Donating/Methanol Accepting" Platform for Preparation of Highly Selective Nafion-based Proton Exchange Membranes. J. Mater. Chem. A 2015, $3,18546-18556$.

(3) Fieldson, G. T.; Barbari, T. A. The Use of FTIR-ATR Spectroscopy to Characterize Penetrant Diffusion in Polymers. Polymer 1993, 34, 1146-1153.

(4) Lai, H. J.; Wang, Z. W.; Wu, P. Y.; Chaudhary, B. I.; Sengupta, S. S.; Cogen, J. M.; Li, B. Structure and Diffusion Behavior of Trioctyl Trimellitate (TOTM) in PVC Film Studied by ATR-IR Spectroscopy. Ind. Eng. Chem. Res. 2012, 51, 9365-9375.

(5) Li, N.; Leng, Y.; Hickner, M. A.; Wang, C.-Y. Highly Stable, Anion Conductive, Comb-shaped 
Copolymers for Alkaline Fuel Cells. J. Am. Chem. Soc. 2013, 135, 10124-10133.

(6) Bae, B. C.; Ha, H. Y.; Kim, D. Preparation and Characterization of Nafion/Poly(1-vinylimidazole) Composite Membrane for Direct Methanol Fuel Cell Application. $J$. Electrochem. Soc. 2005, 152, A1366-A1372.

(7) Sen, U.; Celik, S. U.; Ata, A.; Bozkurt, A. Anhydrous Proton Conducting Membranes for PEM Fuel Cells Based on Nafion/Azole Composites. Int. J. Hydrogen Energy 2008, 33, 2808-2815.

(8) Li, Q.; Li, W.; Zhang, H. N.; Pan, M. Immobilization of Imidazole in Polymer Electrolyte Membranes for Elevated Temperature Anhydrous Applications. J. Appl. Polym. Sci. 2012, 123, 382-387.

(9) Wang, J.; Yue, X.; Zhang, Z.; Yang, Z.; Li, Y.; Zhang, H.; Yang, X.; Wu, H.; Jiang, Z. Enhancement of Proton Conduction at Low Humidity by Incorporating Imidazole Microcapsules into Polymer Electrolyte Membranes. Adv. Funct. Mater. 2012, 22, 4539-4546.

(10) Zhang, F.; Tu, Z.; Yu, J.; Li, H.; Huang, C.; Zhang, H. Impregnation of Imidazole Functionalized Polyhedral Oligomeric Silsesquioxane in Polymer Electrolyte Membrane for Elevated Temperature Fuel Cells. RSC Adv. 2013, 3, 5438-5446.

(11) Song, M.-K.; Li, H.; Li, J.; Zhao, D.; Wang, J.; Liu, M. Tetrazole-based, Anhydrous Proton Exchange Membranes for Fuel Cells. Adv. Mater. 2014, 26, 1277-1282.

(12) Lei, M.; Wang, Y. G.; Zhang, F. F.; Huang, C.; Xu, X.; Zhang, R.; Fan, D. Y. Anhydrous Proton Conducting Composite Membranes Containing Nafion and Triazole Modified POSS. Electrochim. Acta 2014, 149, 206-211.

(13) Tohidian, M.; Ghaffarian, S. R.; Nouri, M.; Jaafarnia, E.; Haghighi, A. H. Polyelectrolyte Nanocomposite Membranes Using Imidazole-functionalized Nanosilica for Fuel Cell Applications. $J$. Macromol. Sci. Part B-Phys. 2015, 54, 17-31.

(14) Amiinu, I. S.; Li, W.; Wang, G.; Tu, Z.; Tang, H.; Pan, M.; Zhang, H. Toward Anhydrous Proton Conductivity Based on Imidazole Functionalized Mesoporous Silica/Nafion Composite Membranes. Electrochim. Acta 2015, 160, 185-194.

(15) Liu, S.; Liu, Y.; Sang, S.; Zhong, W.; Wu, Q. Imidazole/( $\left.\mathrm{HPO}_{3}\right)(3)$-doped Sulfonated Poly(ether ether ketone) Composite Membrane for Fuel Cells. J. Appl. Polym. Sci. 2015, 132, 41946.

(16) Zhang, H.; Wu, W.; Wang, J.; Zhang, T.; Shi, B.; Liu, J.; Cao, S. Enhanced Anhydrous Proton Conductivity of Polymer Electrolyte Membrane Enabled by Facile Ionic Liquid-based Hoping Pathways. J. Membr. Sci. 2015, 476, 136-147.

(17) Sinirlioglu, D.; Muftuoglu, A. E.; Bozkurt, A. Preparation of Thin Films from New Azolic Copolymers and Investigation of Their Membrane Properties. J. Macromol. Sci. Part A-Pure Appl. Chem. 2014, 51, 420-434.

(18) Wu, H.; Cao, Y.; Shen, X.; Li, Z.; Xu, T.; Jiang, Z. Preparation and Performance of Different Amino Acids Functionalized Titania-embedded Sulfonated Poly(ether ether ketone) Hybrid Membranes for Direct Methanol Fuel Cells. J. Membr. Sci. 2014, 463, 134-144.

(19) Lee, C. H.; Lee, Y. M. Highly Proton-conductive Thermally Rearranged Polybenzoxazole for Medium-temperature and Low-humidity Polymer Electrolyte Fuel Cells. J. Power Sources 2014, 247, 286-293.

(20) Hasani-Sadrabadi, M. M.; Dashtimoghadam, E.; Majedi, F. S.; Moaddel, H.; Bertsch, A.; Renaud, P. Superacid-doped Polybenzimidazole-decorated Carbon Nanotubes: A Novel High-performance Proton Exchange Nanocomposite Membrane. Nanoscale 2013, 5, 11710-11717.

(21) Boroglu, M. S.; Celik, S. U.; Boz, I.; Bozkurt, A. Sulfonated Poly(vinyl alcohol)/Triazole 
Blends as Anhydrous Proton Conducting Membranes for Polymer Electrolyte Membrane Fuel Cells. J. Mater. Res. 2013, 28, 1458-1465.

(22) Asgari, M. S.; Nikazar, M.; Molla-abbasi, P.; Hasani-Sadrabadi, M. M. Nafion(R)/Histidine Functionalized Carbon Nanotube: High-performance Fuel Cell Membranes. Int. J. Hydrogen Energy 2013, 38, 5894-5902.

(23) Hu, J.; Luo, J.; Wagner, P.; Agert, C.; Conrad, O. Thermal Behaviours and Single Cell Performance of PBI-OO/PFSA Blend Membranes Composited with Lewis Acid Nanoparticles for Intermediate Temperature DMFC Application. Fuel Cells 2011, 11, 756-763.

(24) Celik, S. U.; Bozkurt, A. Proton Conduction Promoted by 1H-1,2,3-benzotriazole in Non-humidified Polymer Membranes. Electrochim. Acta 2011, 56, 5961-5965.

(25) Ding, Z.; Kim, D. S.; Labouriau, A.; Kim, Y. S. Synthesis of Benzimidazole-tethered Perfluoropolymer/Perfluoromacromolecules for High Temperature Fuel Cell Applications. ECS Trans. 2010, 33, 659-671.

(26) Grandi, S.; Mustarelli, P.; Carollo, A.; Tomasi, C.; Quartarone, E.; Magistris, A. New Fillers for PBI-based Composite Electrolytes in Polymer Fuel Cells. Compos. Interfaces 2010, 17, 649-662.

(27) Quartarone, E.; Magistris, A.; Mustarelli, P.; Grandi, S.; Carollo, A.; Zukowska, G. Z.; Garbarczyk, J. E.; Nowinski, J. L.; Gerbaldi, C.; Bodoardo, S. Pyridine-based PBI Composite Membranes for PEMFCs. Fuel Cells 2009, 9, 349-355.

(28) Hudiono, Y.; Choi, S.; Shu, S.; Koros, W. J.; Tsapatsis, M.; Nair, S.; Porous Layered Oxide/Nafion(R) Nanocomposite Membranes for Direct Methanol Fuel Cell Applications. Microporous Mesoporous Mater. 2009, 118, 427-434.

(29) Aslan, A.; Bozkurt, A. Development and Characterization of Polymer Electrolyte Membranes Based on Ionical Cross-linked Poly(1-vinyl-1,2,4 triazole) and Poly(vinylphosphonic acid). J. Power Sources 2009, 191, 442-447.

(30) Quartarone, E.; Mustarelli, P.; Carollo, A.; Grandi, S.; Magistris, A.; Gerbaldi, C. PBI Composite and Nanocomposite Membranes for PEMFCs: the Role of the Filler. Fuel Cells 2009, 9, 231-236.

(31) Fu, Y. Z.; Manthiram, A. Nafion-Imidazole- $\mathrm{H}_{3} \mathrm{PO}_{4}$ Composite Membranes for Proton Exchange Membrane Fuel Cells. J. Electrochem. Soc. 2007, 154, B8-B12.

(32) Kim, J.-D.; Mori, T.; Hayashi, S.; Honma, I. Anhydrous Proton-conducting Properties of Nafion-1,2,4-triazole and Nafion-Benzimidazole Membranes for Polymer Electrolyte Fuel Cells. $J$. Electrochem. Soc. 2007, 154, A290-A294.

(33) Yamada, M.; Honma, I. Anhydrous Proton Conducting Polymer Electrolytes Based on Poly(vinylphosphonic acid)-Heterocycle Composite Material. Polymer 2005, 46, 2986-2992.

(34) Je-Deok, K.; Suzuki, A.; Jun, M.-S. Nafion-Azole- $\mathrm{H}_{3} \mathrm{PO}_{4}$ Composite Membranes Using Solution Processing for High Temperature PEMFCs. ECS Trans. 2012, 58, 1185-1194. 